Documentation et bibliothèques

\title{
Portraits d'enfance, portraits d'adolescence : pratiques de lecture et pratiques culturelles à travers les étapes du développement humain
}

\section{Geneviève Gareau}

Volume 66, numéro 2, avril-juin 2020

Les publics : mieux les intégrer

URI : https://id.erudit.org/iderudit/1069968ar

DOI : https://doi.org/10.7202/1069968ar

Aller au sommaire du numéro

\section{Éditeur(s)}

Association pour l'avancement des sciences et des techniques de la documentation (ASTED)

\section{ISSN}

0315-2340 (imprimé)

2291-8949 (numérique)

Découvrir la revue

\section{Citer cet article}

Gareau, G. (2020). Portraits d'enfance, portraits d'adolescence : pratiques de lecture et pratiques culturelles à travers les étapes du développement humain. Documentation et bibliothèques, 66(2), 20-30. https://doi.org/10.7202/1069968ar

\section{Résumé de l'article}

L'enfance, l'adolescence et même la préadolescence sont des moments de vie particuliers qui sont marqués, selon certaines recherches, par des pratiques de lecture et des pratiques d'activités technologiques propres à ces âges. En amont de ces pratiques, il reste le lecteur, le jeune, la personne qui vit des changements psychologiques et physiologiques propres à son étape de développement. Ces moments de changements influencent les besoins, les intérêts et les goûts en matière de lecture de ces jeunes. Connaître leurs étapes de développement et leurs besoins, c'est comprendre davantage le public des jeunes, prendre conscience de l'importance de l'étape de développement de ces usagers comme personnes et ainsi avoir la possibilité d'interagir plus profondément et dynamiquement avec eux.

À partir d'études statistiques sur les pratiques de lecture des enfants, des préadolescents et des adolescents, cet article présente ces pratiques en lien avec les étapes de développement reliées à l'âge. Une meilleure connaissance théorique des dimensions physiologique, cognitive, affective et sociale des jeunes permettra un fondement plus fin de nos pratiques académiques et professionnelles dans nos interventions auprès des jeunes.
Tous droits réservés (c) Association pour l'avancement des sciences et des techniques de la documentation (ASTED), 2020
Ce document est protégé par la loi sur le droit d'auteur. L'utilisation des services d'Érudit (y compris la reproduction) est assujettie à sa politique d'utilisation que vous pouvez consulter en ligne.

https://apropos.erudit.org/fr/usagers/politique-dutilisation/ 


\section{PORTRAITS D'ENFANCE, PORTRAITS}

\section{D'ADOLESCENCE: PRATIQUES DE LECTURE ET PRATIQUES CULTURELLES À TRAVERS LES ÉTAPES DU DÉVELOPPEMENT HUMAIN}

Geneviève GAREAU

M.S.I., B.A.A., candidate au doctorat en sciences de l'information École de bibliothéconomie et des sciences de l'information, Université de Montréal genevieve.gareau@umontreal.ca

\begin{abstract}
L'enfance, l'adolescence et même la préadolescence sont des moments de vie particuliers qui sont marqués, selon certaines recherches, par des pratiques de lecture et des pratiques d'activités technologiques propres à ces âges. En amont de ces pratiques, il reste le lecteur, le jeune, la personne qui vit des changements psychologiques et physiologiques propres à son étape de développement. Ces moments de changements influencent les besoins, les intérêts et les goûts en matière de lecture de ces jeunes. Connaître leurs étapes de développement et leurs besoins, c'est comprendre davantage le public des jeunes, prendre conscience de l'importance de l'étape de développement de ces usagers comme personnes et ainsi avoir la possibilité d'interagir plus profondément et dynamiquement avec eux. À partir d'études statistiques sur les pratiques de lecture des enfants, des préadolescents et des adolescents, cet article présente ces pratiques en lien avec les étapes de développement reliées à l'âge. Une meilleure connaissance théorique des dimensions physiologique, cognitive, affective et sociale des jeunes permettra un fondement plus fin de nos pratiques académiques et professionnelles dans nos interventions auprès des jeunes.
\end{abstract}

Childhood, adolescence and even preadolescence are moments of life that are scarred, according to some research, by reading and technological practices specific to these age groups. Among these practices remains the reader, the young, the person who lives the psychological and physiological changes specific to his development stage. These moments of change influence the reading needs, interests and tastes of this youth. To know their development stages and their needs, is to understand the readership of the youth, to acknowledge the importance of the development step of these users as people and therefore to have the possibility to interact in a more profound and dynamic way with them.

Based on statistical studies on children, preadolescents and adolescents' reading practices, this article presents those practices, tied with the development stages linked to age. A better theoretic knowledge of the physiological, cognitive, emotional and social dimensions of the youth will enable a more refined foundation of our academic and professional practices with intervening with the youth.

\section{Introduction}

Thématique actuelle, la connaissance des publics est essentielle pour penser les bibliothèques comme des communautés par le biais de l'inclusion, de l'attention à la personne, etc., permettant ainsi de favoriser l'émergence d'un espace social commun et collaboratif. Design thinking, design du care, modèles et philosophie de codesign permettent l'avancée de ces idées, mais surtout leur concrétisation en conceptualisant et en repensant l'institution qu'est la bibliothèque dans une toute nouvelle essence (Martel, 2017). Au cœur de ces méthodes, la participation des citoyens dans les démarches de design permet de connaitre et de considérer

les besoins et les goûts des publics desservis dans la conception de ces nouvelles bibliothèques (Martel, 2017).

Inclure les publics dans le projet de codesign, connaître les usagers ou encore les publics desservis, c'est également pouvoir situer une personne dans son cycle de vie, soit connaître et savoir reconnaître les caractéristiques liées aux étapes de vie de la personne. Chaque moment de vie est caractérisé par des besoins, des changements qui sont reliés à la fois à un stade de développement et à l'existence humaine, c'est-à-dire que chaque personne traversera ces périodes de développement et le fera à sa façon. Nourrisson, enfant, adolescent, jeune adulte, adulte: à travers chacun de ces stades de vie, nous vivons différents évènements 
et nous traversons des changements; il y a une évolution de notre personne.

Afin de mieux connaître l'enfant-lecteur et l'ado-lecteur dans leurs goûts et leurs besoins en matière de lecture, nous proposons dans cet article de tracer et de brosser un portrait de l'enfance dans son passage à l'adolescence. Moment important et sensible dans la vie d'une personne, cette période de transition est marquée par de nombreux changements aux niveaux physique, cognitif et affectif ainsi que dans la façon de socialiser. Des changements sont même perçus, selon certaines recherches, dans les activités de lecture selon l'âge de la personne (McGeown, Osborne, Warhurst, Norgate et Duncan, 2016; Poirier et al., 2012; Reuter, 2007). Par exemple, la période associée à l'arrivée au secondaire peut s'accompagner d'un désengagement et d'une diminution de l'intérêt pour la lecture qui se traduit par une baisse de la fréquence de lecture.

Connaître les étapes et les stades développement de l'enfant et de l'adolescent est important, car cela permet afin de comprendre davantage l'enfant-lecteur et l'ado-lecteur, la nature des relations qu'ils entretiennent avec le monde ainsi que la constitution de leur petit monde à eux.

Nous décrirons la période correspondant à la fin de l'enfance ainsi que les périodes de début et de fin de l'adolescence en commençant par montrer un portrait de leur pratique de lecture. Nous proposons également d'établir une distinction entre le concept de «pratiques» et celui de «comportement». Dans les recherches et les études statistiques portant sur la lecture, ces concepts sont parfois utilisés de façon indifférenciée pour faire référence aux activités de lecture. Pourtant, ces concepts portent des définitions et des significations distinctes : il est donc important de bien les définir afin de bien les utiliser pour avoir des portraits et une analyse reflétant le plus possible la réalité. Ensuite, nous présenterons une description de l'ensemble des dimensions de la personne: des états affectifs, le point de vue moral, l'évolution psychologique ainsi que la conception et la façon d'établir la relation aux autres. Finalement, nous ferons une mise en rapport de ces portraits et des changements observés entre les caractéristiques de l'enfance et de l'adolescence.

\section{Méthodologie}

Les définitions de pratiques et de comportement proposées dans les prochaines sections proviennent d'une revue de littérature effectuée dans le cadre de travaux pour alimenter notre recherche doctorale. Comme corpus de travail, nous avons utilisé des articles scientifiques en sciences de l'information (SI), des articles provenant d'encyclopédies spécialisées, des études statistiques ainsi que des ouvrages en psychologie traitant du développement humain.
Pour relever les articles scientifiques en SI portant sur les pratiques de lecture, nous avons utilisé la base de données LISA avec la requête ( reading practice ${ }^{*}$ » $O R$ « pratique ${ }^{*}$ de lecture»), qui a retourné 19 articles disponibles en anglais ou en français. Pour les articles scientifiques sur les comportements en lecture, nous avons utilisé la même base de données avec, cette fois, la requête suivante: ("comportement* en lecture» OR «comportement* de lecture» $O R$ «reading behavior* ${ }^{*}$ OR «reading behaviour*»). Cette requête a retourné 23 articles disponibles en anglais ou en français.

Les études statistiques disponibles portant sur les activités de lecture des enfants et des adolescents ont été choisies à la suite d'une recherche sur Google. Deux études québécoises, une canadienne et deux françaises ont été sélectionnées.

Les informations concernant les stades de développement proviennent d'ouvrages (parus entre 2006 et 2017) en psychologie. Ils ont été relevés à partir du catalogue Atrium des bibliothèques de l'Université de Montréal en utilisant les termes «Enfants - développement» et «Adolescents développement» dans l'index par sujet.

Dans la prochaine section, nous présenterons des définitions des termes «pratiques» et "comportement» utilisés dans la recherche en SI, en sociologie et en psychologie.

\section{Lecture, comme des pratiques ou comme un comportement: une distinction par la définition}

Quelle(s) différence(s) peut-il exister entre pratiques de lecture et comportement de lecture? Avant de commencer la présentation du portrait de l'enfant-lecteur et de l'adolecteur, il est important de poser et de clarifier la signification des concepts de "pratiques» et de "comportement», et ce, en le faisant en tenant compte du contexte où se déroulent nos réflexions, c'est-à-dire la lecture. Pour mener à bien notre analyse, le fait d'établir des définitions des concepts de «pratiques» et de «comportement» nous permettra d'encadrer leur utilisation à travers nos réflexions et ainsi de garantir une uniformité dans le traitement et la présentation de ces concepts.

À la suite d'une revue de la littérature, il a été possible de relever l'utilisation faite des termes de "pratiques» et de «comportement» dans des études statistiques et dans la littérature scientifique en SI portant sur la lecture. Nous sommes arrivés aux trois constats suivants. Premièrement, il nous a été possible de relever que, dans la littérature scientifique en SI portant sur la lecture, ces notions, bien que très présentes et utilisées, sont peu et même rarement définies avec détails et précision (ex.: Ashfaq et Ansari, 2018; Burleigh, 2017; Choi et al., 2017; Dawkins, 2017; 
Kleijnen, Huysmans, Ligtvoet et Elbers, 2017; Late, 2018; Rutherford et al., 2017). Il est possible de constater également que ces termes sont utilisés indistinctement dans un même texte ou article, comme des synonymes. De manière plus large, les concepts de comportement et de pratiques sont plutôt utilisés en SI dans des contextes de recherche informationnelle et sont repris dans plusieurs modèles conceptuels largement utilisés dans le domaine (ex.: Ellis, 1989; Jansen et Rieh, 2010; Savolainen, 2007, 2019; Wilson, 1999). Deuxièmement, toujours à la suite d'une revue de la littérature sur la lecture en sciences de l'information sur l'utilisation des termes de "pratiques» et de "comportement», nous avons constaté que le terme "pratiques» était davantage employé dans le domaine sociologique, écrit sous sa forme plurielle, et que le terme "comportement» était davantage utilisé dans le domaine psychologique (ex. : Burleigh, 2017; Kleijnen et al., 2017 ; Late, 2018; Lopatovska et Sessions, 2016; Rutherford et al., 2017). Finalement, dans le contexte de la recherche sur la lecture dans le domaine des SI, la lecture est également présentée, dans un point de vue de la sociologie de la lecture, comme une pratique culturelle.

Nous présentons dans les prochaines sous-sections l'utilisation de «pratique informationnelle» et de «comportement informationnel » en SI, une définition du terme " pratiques » $\mathrm{du}$ point de vue sociologique et une définition du terme «comportement» du point de vue psychologique ainsi qu'une description de ce que peut être une pratique culturelle.

\section{Pratique informationnelle ou comportement informationnel}

La littérature en SI s'est penchée sur le comportement informationnel (information behavior), mais également sur la pratique informationnelle (information practice). Ces deux concepts sont définis dans la littérature scientifique en SI, à la base, comme des activités humaines (Fulton et Henefer, 2017, p. 2163). Ces termes sont cependant utilisés différemment puisqu'ils réfèrent chacun, comme concept, à des perspectives théoriques différentes (Savolainen, 2007, p. 110). Ils ne sont donc ni interchangeables ni synonymes.

Le concept de «comportement informationnel» se définit selon les aspects cognitifs, les aspects psychologiques, les actions et les habitudes de l'individu qui adopte le comportement (Fulton et Henefer, 2017, p. 2162; Savolainen, 2007, p. 119). Ce concept se rattache davantage au domaine de la psychologie. En SI, Wilson (1999) définit le comportement informationnel comme étant «les activités effectuées par une personne lorsqu'elle identifie ses propres besoins en information, lorsqu'elle recherche cette information et lorsqu'elle utilise ou transfère cette information » [traduction libre] (p. 249). Dans ce modèle de Wilson, le comportement informationnel devient l'ensemble le plus général et englobe les sous-ensembles du comportement de recherche d'information et de repérage d'information (Wilson, 1999, p. 263). Le comportement de recherche d'information renvoie à la variété des méthodes utilisées pour trouver des sources d'information et y accéder. Le comportement en repérage d'information représente les interactions entre les utilisateurs et les systèmes d'information.

Le concept de «pratique informationnelle» est un concept plus large qui couvre le concept de comportement informationnel et qui s'associe au domaine de la sociologie (Fulton et Henefer, 2017, p. 2162). Le concept de "pratique» est utilisé soit comme un concept théorique, soit en référence à une activité reliée à une expérience (Fulton et Henefer, 2017, p. 2163). Fulton et Henefer (2017) définissent le concept de «pratique» comme le fait, pour un individu, de s'approprier une coutume ou une tradition en la concrétisant dans une activité qui peut devenir une routine ou une habitude (p. 2163).

Le concept de «lecture» pourrait être associé à un comportement informationnel, mais peut montrer et présenter une notion plus large que le comportement informationnel ou la pratique d'information. Employées également dans les domaines de la sociologie et de la psychologie, l'utilisation et la référence à des définitions de «pratiques » et de «comportement» provenant de ces deux domaines permettraient d'avoir une compréhension plus fine et plus précise de ces deux concepts. Ces ajouts disciplinaires aux définitions bibliothéconomiques de "pratiques» et "comportement» permettraient, selon notre optique, une utilisation plus juste des termes dans la pratique tant professionnelle qu'académique et, éventuellement, de faire une mesure plus précise de ces concepts et ainsi d'augmenter la valeur de validité des résultats.

Nous présentons dans la prochaine section les définitions des termes «pratiques» et «comportement» du point de vue de la sociologie et de la psychologie.

\section{Définition du terme pratiques du point de vue sociologique}

La perspective sociologique permet d'aborder les connaissances du point de vue de la réalité sociale, des individus qui la composent et des relations que les individus tissent entre eux (Campeau, Sirois et Rheault, 2004, p. 16; Godefroid, 2008, p. 10). Cette perspective permet de comprendre l'acte individuel d'un point de vue global, c'està-dire selon l'ensemble des activités des acteurs sociaux (Campeau et al., 2004, p. 16; Godefroid, 2008, p. 10). Le concept de «pratiques » peut également être défini comme «un portrait de l'activité humaine dans son contexte culturel et institutionnel» [traduction libre] (Biernacki, 2007). 
La définition de «pratiques» peut également être vue comme «une logique et des comportements spécifiques à chaque milieu » (Ferréol, Cauche, Duprez, Gadrey et Simon, 2011, p. 220). En effet, dans la théorie de la pratique bourdieusienne, l'acte individuel a un fondement sociologique puisqu'il est aussi déterminé par la structure sociale, composée entre autres de la classe et de la position sociales, dont fait partie la personne (Eisenberg, 2007; Fortier et Pizarro Noël, 2013, p. 51). Les goûts, les préférences et les manières d'être sont parfois le reflet de la classe et de la position sociales auxquelles l'individu appartient et sont transmis par l'éducation (Fortier et Pizarro Noël, 2013, p. 51). Cette reproduction des distinctions de goûts et de manières entre les classes sociales est représentée, chez Bourdieu, par le concept d' «habitus» (Eisenberg, 2007). Ce concept se définit comme l'ensemble des savoirs, des savoirfaire et des savoir-être transmis à l'individu et qui, intégrés par lui, se concrétisent par des pratiques, c'est-à-dire par des conduites et des préférences (Fortier et Pizarro Noël, 2013, p. 51). Dans le cas de la lecture, ce sont effectivement principalement les parents qui transmettent à leurs enfants le goût de la lecture ainsi que les codes nécessaires pour conduire une lecture (McKechnie, 2006, p. 73-75). Par cette transmission, les parents permettent à leurs enfants d'intégrer ou non la grande communauté des lecteurs (McKechnie, 2006, p. 68 ; Ross, 2006, p. 224 ; Rothbauer, 2006, p. 127).

\section{Définition du terme comportement du point de vue psychologique}

La perspective psychologique permet d'évaluer «l'interaction des facteurs qui influencent le fonctionnement de la personne» afin de comprendre comment un comportement particulier peut être adopté par celle-ci (Campeau et al., 2004, p. 16; Godefroid, 2008, p. 18-19).

Dans le domaine de la psychologie, le «comportement» se définit comme étant tout ce qui est objectivement observable, incluant «les activités de l'esprit» (Fröhlich, 1997, p. 84). La notion de "comportement» est également vu comme une «manière d'être et d'agir» des êtres humains et des «manifestations objectives de leur activité globale» (Bloch, 1997, p. 240). Par "observable», il est entendu que le comportement puisse être mesuré par l'observation scientifique afin de recueillir des faits, et non pas par une mesure interne, c'est-à-dire selon le point de vue du sujet lui-même par le biais de l'entrevue, par exemple (Bloch, 1997, p. 240).

\section{Lecture comme pratiques culturelles}

Du point de vue de la sociologie de la lecture, les «pratiques culturelles» peuvent être définies comme étant «l'ensemble des activités de consommation ou de participation liées à la vie intellectuelle et artistique, qui engagent des dispositions esthétiques et participent à la définition des styles de vie: lecture, fréquentation des équipements culturels [théâtres, musées, salles de cinéma, salles de concert, etc.], usages des médias audiovisuels, mais aussi pratiques culturelles amateurs» (Coulangeon, 2010, p. 3-4). Ces pratiques regroupent un ensemble d'activités dont la variété dépendrait des pays et des époques dans lesquels elles se situent (Coulangeon, 2010, p. 4). Pour Coulangeon (2010, p. 5), la compréhension et la connaissance des habitudes des pratiques culturelles sont essentielles puisque ces habitudes sont généralement le reflet de la stratification sociale et, donc, des inégalités sociales. Ces études sur les pratiques culturelles peuvent mener, entre autres, à établir les politiques publiques sur la culture afin de contribuer à atténuer ces inégalités sociales.

Dans le cadre de cet article, nous proposons de brosser un portrait de l'enfant-lecteur et de l'ado-lecteur en présentant les activités de lecture effectuées en général à ces âges, tout en décrivant en parallèle le stade de développement de l'enfance et de l'adolescence. Nous cherchons donc à comprendre les activités de lecture reliées à deux groupes de personnes en particulier, soit les enfants et les adolescents du Québec. De la même façon, nous ne cherchons ni à comprendre les activités de lecture d'un individu ni à comprendre les facteurs et le contexte qui peuvent ou pourraient influencer ces activités. L'utilisation du concept de «pratiques» serait donc plus appropriée dans le soutien de la présente analyse et dans le cadre particulier de cet article.

Le concept de «pratiques de lecture» pourrait ainsi se définir comme étant un portrait général de la tendance des activités de lecture effectuées par un groupe d'acteurs sociaux situé dans une société en particulier.

Dans la prochaine section, nous ferons un portrait des pratiques de lecture de ces jeunes accompagnés d'un bref portrait de la population des jeunes au Québec.

\section{Pratiques de lecture}

Au Québec, la population des 10-14 ans et des 15-19 ans reste relativement stable depuis 2010. Les 10 à 14 ans représentent environ $5 \%$ de la population depuis les dix dernières années et en représentent 5,2\% exactement en 2019 (Gouvernement du Québec, 2019). Les 15 à 19 ans constituent autour de $6 \%$ de la population depuis les dix dernières années, et 5,93\% en 2019 (Gouvernement du Québec, 2019).

Entre 2004 et 2014 au Québec, la proportion de personnes adultes affirmant avoir lu au moins un livre dans l'année est passée de $84,1 \%$ à 80,2\% (Magnan, 2016, p. 39 ; Vachon, 2010, p. 26). Cette tendance à la baisse du nombre 
de livres par année se remarque également chez les enfants, particulièrement au moment du passage du primaire au secondaire.

$\mathrm{Au}$ Canada, la régularité dans la fréquence des activités de lecture et la moyenne du nombre de livres lus par année diminuent lorsque les enfants avancent en âge (Scholastic, 2017). En 2017, 50 \% des 6 à 8 ans ont déclaré lire régulièrement, soit de 5 à 7 fois par semaine, alors que le nombre de lecteurs dits réguliers va baisser à $37 \%$ chez les 9 à 11 ans et à $24 \%$ chez les 12 à 14 ans pour revenir à $25 \%$ chez les 15 à 17 ans (Scholastic, 2017). La moyenne des livres lus en 2017 va passer de 37 livres pour les 6 à 8 ans à 23 livres pour les 9 à 11 ans, à 16 livres pour les 12 à 14 ans et, finalement, à 15 livres pour les 15 à 17 ans (Scholastic, 2017). Fait à noter, le Québec est la province où, en 2017, le nombre moyen de livres lus est le plus bas chez les enfants, soit de 19 livres par année comparativement à 30 livres en Alberta, qui est la province montrant le plus haut nombre de livres lus au Canada, et à 23 livres pour la moyenne canadienne (Scholastic, 2017).

Il y a également un effet d'âge par rapport au plaisir de lire. Ainsi, au Canada, alors que $37 \%$ des enfants de 6 à 8 ans s'adonnent à la lecture par plaisir, seuls $34 \%$ des 9 à 11 ans disent lire par plaisir, et $24 \%$ des 12 à 14 ans et $26 \%$ des 15 à 17 ans le font (Scholastic, 2017). Cependant, cette tendance à la baisse de la fréquence de lecture ne signifie pas nécessairement que les enfants n'apprécient pas ou apprécient moins la lecture. En effet, bien que seulement $37 \%$ des enfants de 9 à 12 ans au Canada lisent régulièrement, $77 \%$ affirment aimer lire par plaisir (Scholastic, 2017). Il est d'ailleurs possible de remarquer que les jeunes plus âgés semblent avoir des difficultés à trouver des livres qui correspondent à leurs goûts et envies de lecture, particulièrement pour les 12 à 14 ans qui affirment, dans $55 \%$ des cas, avoir du mal à trouver de la lecture qui leur plaît (Scholastic, 2017).

On remarque aussi que, plus les enfants gagnent en âge, plus ils sont susceptibles de choisir des activités incluant des technologies numériques pendant leur temps libre (Scholastic, 2017). Plus les jeunes vieillissent, plus la navigation sur Internet, le visionnement de YouTube, le fait de jouer à des jeux électroniques ainsi que l'utilisation d'un appareil cellulaire et de médias sociaux sont en hausse. Pour la navigation sur Internet, $27 \%$ des enfants de 6 à 8 ans disent naviguer sur le web 5 à 7 fois par semaine, pour atteindre $32 \%$ chez les 9 à 11 ans et $58 \%$ pour les 12 à 14 ans (Scholastic, 2017). $36 \%$ des 9 à 11 ans disent visionner des vidéos YouTube 5 à 7 fois par semaine et le pourcentage va atteindre les $54 \%$ et $53 \%$ chez les 12 à 14 ans et chez les 15 à 17 ans (Scholastic, 2017). Les jeux électroniques augmentent en popularité : $41 \%$ des jeunes entre 9 à 11 ans s'y adonnent 5 à 7 fois par semaine pour passer à $52 \%$ de 12 à 14 ans (Scholastic, 2017). Les 15 à 17 ans sont de grands consommateurs de leur téléphone cellulaire puisque $68 \%$ d'entre eux l'utilisent de 5 à 7 fois par semaine, comparativement à $49 \%$ des 12 à 14 ans et à seulement $18 \%$ des 9 à 11 ans (Scholastic, 2017). La même tendance se dessine pour la consultation des médias sociaux: $66 \%$ des 15 à 17 ans disent les regarder de 5 à 7 fois par semaine alors que $43 \%$ des 12 à 14 ans s'y adonnent et que seuls $14 \%$ des 9 à 11 ans affirment regarder les réseaux sociaux (Scholastic, 2017).

Ces jeunes côtoient de manière quotidienne les technologies et l'informatique, et ce, dans plusieurs sphères de leur vie, comme à l'école, par l'utilisation des tableaux blancs interactifs et d'applications pédagogiques ou de manuels scolaires sur des tablettes numériques ou des ordinateurs. Dans leurs activités de loisirs, près des deux tiers des jeunes de 12 à 17 ans passent 15 heures ou plus par semaine devant des écrans (Du Mays et Bordeleau, 2015). Pourtant, il semble que la lecture sur support numérique soit une pratique peu adoptée par les enfants au Québec. En effet, dans une étude produite par Scholastic (2017), $84 \%$ des enfants de 6 à 11 ans affirment préférer le format papier au format numérique, même si le matériel de lecture est disponible dans les deux formats. Le ratio diminue à $76 \%$ pour les jeunes de 12 à 17 ans. De plus, les prêts des enfants représentaient seulement $7,5 \%$ des prêts numériques en bibliothèques publiques en 2016 (Bibliopresto.ca, 2017, p. 10) et ont connu une légère augmentation à 7,86\% en 2017 et à 7,96\% en 2018 (Bibliopresto.ca, 2018, p. 13).

Afin de mieux comprendre les besoins de ces jeunes, nous présenterons, dans les prochaines sections, une description de ce que sont les stades de développement, ainsi qu'une description des caractéristiques des stades de développements de l'âge de l'enfance et celui de l'adolescence.

\section{Stade de développement}

Tout au cours du cycle de vie, une personne évoluera sur les plans moral, cognitif, éthique, physique, social, etc. La personne verra également ses comportements et ses besoins se modifier et évoluer tout au cours de son cycle de vie. Cette évolution est propre à l'espèce humaine, mais aussi à chaque être humain, et elle suit une séquence d'étapes qui sont appelées, dans le domaine de la psychologie, «stades de développement». Ce découpage du cycle de vie en stades de développement humain permet de comprendre et de décrire les différents processus et changements qui s'opèrent pendant la vie d'une personne et qui correspondent à des étapes particulières dans l'existence humaine (Demont, 2009, p. 63; Papalia, Olds et Feldman, 2010, p. 4-5). Ainsi, le développement de l'être humain est constitué de changements, principalement situés pendant la petite enfance, mais est également fait de grandes périodes 
de continuité, pendant son âge adulte par exemple (Papalia et al., 2010, p. 5). L'évolution de la personne se poursuit donc tout au long de l'existence, allant de la conception jusqu'à la mort (Demont, 2009, p. 64). Le cycle de vie est découpé en quatre grandes phases présentant chacune des caractéristiques particulières: la période prénatale, l'âge de l'enfance, l'âge de l'adolescence et l'âge adulte.

Les stades de développement touchent toutes les dimensions rattachées à la personne. Les processus et les changements constatés d'un stade à l'autre peuvent affecter les différentes dimensions de la personne telles que les dimensions physiologique, cognitive, affective, émotionnelle et sociale (Papalia et al., 2010, p. 2-3). Le développement de la personne est un processus global, dynamique et complexe où chacune des dimensions de la personne, en période de changement ou de stabilité, va interagir avec les autres (Papalia, Olds et Feldman, 2010, p. 4). Par exemple, un changement hormonal peut entraîner des changements sur le plan émotionnel et avoir des répercussions sur le comportement de la personne en société. Ces processus et ces changements sont déterminés par notre nature humaine et sont communs à tous les êtres humains (Papalia et al., 2010, p. 4). Ces changements n'apparaissent pas et ne sont pas conduits par le fruit du hasard, puisqu'il existe des principes et des caractéristiques typiques propres à chaque stade de développement, qui sont remarqués de façon générale dans l'ensemble de la population (Papalia et al., 2010, p. 5; Shaffer et Kipp, 2014, p. 5). En effet, les périodes de maturation du cerveau et du corps suivent des étapes assez fixes selon l'âge de la personne (Papalia et al., 2010, p. 7). Ces changements restent cependant spécifiques à l'individu dans le sens où chacun vit ces changements d'une manière différente, particulière et unique (Papalia et al., 2010, p. 2-3; Shaffer et Kipp, 2014, p. 5). Les expériences vécues par la personne, son milieu et son mode de vie façonnent également ces changements, ce qui explique en partie les différences individuelles qu'il peut y avoir dans le développement d'une personne (Papalia et al., 2010, p. 7). Deux types de développement sont donc remarqués, soit un développement normatif, qui représente le développement «type» qu'il est possible de retrouver à travers les stades de vie de la personne, et un développement idéographique, qui représente les variations par rapport à un développement «type» qu'une personne peut traverser pendant son développement au cours de son existence (Shaffer et Kipp, 2014, p. 5).

Les changements physiologiques correspondent aux changements qui affectent le corps, le cerveau ainsi que les capacités sensorielles et motrices (Papalia et al., 2010, p. 6). Les changements peuvent se faire, notamment, sur les plans de la taille, du poids, de la croissance du cerveau (Papalia et al., 2010, p. 3). Le développement physiologique a particulièrement une influence sur l'intelligence, les facultés intellectuelles et la personnalité (Papalia et al., 2010, p. 6).
Considérée également comme un facteur individuel, la dimension cognitive couvre la façon dont la personne acquiert des connaissances ainsi que la façon dont celles-ci sont organisées par le cerveau (Godefroid, 2008, p. 703). Les changements sont reliés aux habiletés mentales de la personne comme la perception, l'apprentissage, la mémoire, le langage, le raisonnement et la créativité (Papalia et al. 2010 , p. 6). Il peut s'agir de la nature de l'intelligence ou encore du nombre de mots contenus dans le vocabulaire de la personne (Papalia et al., 2010, p. 3).

Le développement affectif concerne les émotions vécues de la personne ainsi que sa personnalité. Le développement affectif de la personne est en lien avec la nature et la qualité des relations qu'elle entretient avec son entourage (Papalia et al., 2010, p. 6). Par exemple, l'évolution de l'attachement aux personnes significatives est considérée comme faisant partie du développement affectif (Papalia et al., 2010, p. 3).

La dimension sociale, quant à elle, fait référence aux liens qu'une personne établit avec les personnes autour d'elle ainsi qu'aux habitudes sociales qu'elle acquiert (Godefroid, 2008, p. 723). La capacité à créer ces liens est déterminée par des mécanismes innés, le développement du système nerveux et les expériences vécues (Godefroid, 2008, p. 723). La relation avec les autres fait partie du développement social (Papalia et al., 2010, p. 6). Le comportement agressif et l'orientation professionnelle sont des exemples de développement social (Papalia et al., 2010, p. 3).

Le tableau 1 liste les différentes dimensions de la personne touchées par le développement de la personne ainsi que les caractéristiques reliées aux dimensions.

\section{TABLEAU 1}

Dimensions de la personne et leurs caractéristiques

\begin{tabular}{ll} 
Dimension de la personne & Caractéristiques de la dimension \\
\hline Physiologique & Corps \\
& Cerveau \\
& Capacités sensorielles \\
& Capacités motrices \\
\hline Cognitive & Habiletés mentales \\
& Perception \\
& Apprentissage \\
& Mémoire \\
& Langage \\
& Raisonnement \\
Créativité \\
\hline Affective & Émotions vécues \\
& Personnalité \\
\hline Sociale & Relation avec les autres \\
& Statut social \\
\hline
\end{tabular}

Papalia et al., 2010, p. 2-7; Shaffer et Kipp, 2014, p. 5 
Au-delà du cheminement type et général que peut prendre le développement humain, celui-ci peut être influencé par des facteurs internes ou externes à la personne (Papalia et al., 2010, p. 8-9). Les facteurs internes à la personne sont l'hérédité, soit le bagage génétique, et la maturation, qui consiste en des changements neurophysiologiques et biochimiques qui permettent à la personne d'acquérir et de maîtriser davantage certaines habiletés (Papalia et Martorell, s. d., p. 8). Les facteurs externes correspondent à l'influence du milieu et de l'environnement comme le milieu familial, la société et la culture dans lesquels elle évolue et grandit. Les relations qu'entretient la personne avec son milieu exercent une influence normative sur son développement par les expériences qu'elle aura vécues avec ses pairs, membres de sa famille, etc. (Papalia et Martorell, s. d., p. 9). Finalement, le développement de l'être humain sera aussi influencé par les moments de vie, des évènements inhabituels ou extraordinaires qui peuvent être heureux ou dramatiques et qui influenceront l'expérience de vie de la personne (Papalia et Martorell, s. d., p. 9).

Dans la prochaine section, nous présentons un portrait des caractéristiques de l'âge de l'enfance à la période de la préadolescence.

\section{L'âge de l'enfance: la période de la préadolescence}

De nombreux changements s'opèrent pendant l'âge de l'enfance: de la naissance à la jeune enfance jusqu'à la préadolescence, la personne poursuit une évolution fulgurante pendant cette période. Pour les fins de cet article, comme nous nous intéressons au passage de l'enfance à l'adolescence, nous nous attarderons à la préadolescence, porte d'entrée vers l'adolescence.

Durant l'enfance, plusieurs changements se vivent du point de vue physique. Pendant la période de la préadolescence, la poussée de croissance continue, mais l'évolution et le développement physique s'effectuent de manière plus régulière comparativement aux changements qui peuvent être observés lors de la petite enfance. Les personnes du genre sexuel féminin sont souvent plus grandes de taille que ceux du genre sexuel masculin à cet âge, tendance qui va généralement se renverser à l'adolescence (Boyd et Bee, 2017, p. 214). Aussi, les premiers signes de la puberté commencent à apparaître (Boyd et Bee, 2017, p. 214). On note particulièrement l'acquisition et le développement de la motricité fine pendant l'adolescence. L'enfant devient plus habile dans l'exécution de certaines tâches et acquiert une plus grande dextérité manuelle (Boyd et Bee, 2017, p. 214). Les mouvements sont également plus précis et plus coordonnés qu'auparavant (Boyd et Bee, 2017, p. 273).
Après avoir maîtrisé le monde symbolique, l'enfant, lors de la préadolescence, continue à se développer cognitivement et commence à utiliser et à appliquer des opérations logiques et concrètes. En explorant le monde extérieur, il comprend qu'une certaine logique régit et gouverne l'ordre des choses (Boyd et Bee, 2017, p. 218). Le préadolescent arrive à manipuler mentalement des objets et à les appliquer dans des opérations mathématiques (Boyd et Bee, 2017, p. 218). Il développe et maîtrise les notions de conservation de poids et de volume, ainsi que la notion de temps et de vitesse (Godefroid, 2008, p. 708). Il arrive aussi à utiliser des outils de mesure. L'acquisition des habiletés reliées aux opérations concrètes est nécessaire pour pouvoir passer à la prochaine étape de développement cognitif que forment les opérations formelles, soit la manipulation mentale d'idées et de concepts (Boyd et Bee, 2017, p. 218-219).

Le développement affectif lors de l'enfance se fait en deux étapes, soit la capacité à exprimer ses émotions puis à comprendre ces émotions. Les émotions jouent un rôle essentiel dans le développement de la personne, puisqu'elles peuvent amener ou motiver à poser un comportement (ex. : ne pas aimer les légumes verts) et peuvent interférer dans le fonctionnement de la personne (ex. : la colère et l'agressivité qui peut s'ensuivre) (Keenan, Evans et Crowley, 2016, p. 246). Le développement affectif va souvent de pair avec le développement cognitif (Keenan et al., 2016, p. 249). Ainsi, dans la période de la petite enfance, les jeunes enfants, avant de communiquer avec des mots, vont utiliser des signes corporels (Keenan et al., 2016, p. 246). Pendant la préadolescence, le spectre des émotions vécues s'élargit et les enfants apprennent à réguler leurs émotions (Boyd et Bee, 2017, p. 245; Keenan et al., 2016, p. 249-250). Les préadolescents expérimentent et comprennent qu'ils peuvent, ainsi que les autres autour de lui, ressentir plusieurs émotions à la fois (Keenan et al., 2016, p. 252). À cette période de la vie, les enfants commencent à apprendre à contrôler et à nuancer l'expression de leurs émotions dans les différentes sphères de leur vie (Keenan et al., 2016, p. 246). Ils sont capables à cet âge de contrôler leurs émotions négatives (Keenan et al., 2016, p. 255). Cette aptitude leur permet également de persévérer dans une activité ou de perdurer dans l'exécution d'une tâche malgré le stress, par exemple (Boyd et Bee, 2017, p. 249-252; Keenan et al., 2016, p. 255).

À la préadolescence, la nature des relations et des interactions avec les pairs se modifie. À cette période, les enfants commencent à découvrir les relations sociales et à interagir avec des groupes à l'extérieur de leurs relations familiales. D'une part, les rapports avec l'école changent: l'enfant développera ou non un goût pour l'implication et l'engagement dans son travail et pour l'exécution des tâches (Godefroid, 2008, p. 739). Certains élèves, par leur relation 
avec le milieu scolaire pendant cette période, seraient susceptibles de développer un sentiment d'infériorité par rapport au travail scolaire (Godefroid, 2008, p. 739). D'autre part, les préadolescents sont de plus en plus intéressés par les autres et à entrer en contact avec eux; leurs groupes d'amis et de connaissances s'élargissent (Keenan et al., 2016, p. 289). Les parents laissent souvent, à cette période, une plus grande liberté d'action à leurs enfants, qui ont moins besoin de supervision comparativement aux besoins de supervision et d'encadrement nécessaires lors de la petite enfance (Keenan et al., 2016, p. 289). Avec un intérêt grandissant pour les autres et un moins grand besoin d'encadrement parental, les préadolescents sont moins absorbés par les activités de la maison, se détachent tranquillement du noyau familial et s'investissent davantage dans des activités sociales, récréatives, sportives et autres (Keenan et al., 2016, p. 289). C'est d'ailleurs à cet âge que les enfants commencent à fréquenter la bibliothèque publique sans la compagnie de leurs parents.

\section{L'âge de l'adolescence}

Âge transitoire entre l'enfance et l'âge adulte, l'adolescence est une période parfois perçue comme plutôt difficile, comme un passage entre deux âges. Balloté entre ces deux moments de vie, l'adolescent vit une période de deuil, c'est-à-dire celui de la nostalgie de la période de l'enfance.

Les périodes d'entrée et de fin de l'adolescence sont marquées individuellement (par des facteurs biologiques, cognitifs et psychiques), juridiquement, socialement et culturellement (Cannard, 2015, p. 26-27). Il n'y a pas d'âge fixe pour «commencer» l'adolescence ou même d'âge fixe qui annonce la fin de cette période. Il s'agit d'une période de vie qui s'est considérablement allongée depuis les dernières décennies, d'une part par la puberté qui arrive de manière beaucoup plus précoce (Coslin, 2017, p. 13) et, d'autre part, par la fin de l'adolescence qui est, de nos jours, de plus en plus tardive (Habib et Cassotti, 2017, p. 383). Si le début de l'adolescence est souvent associé aux changements physiologiques et biologiques, qui sont très visibles, il est plus difficile et complexe de déterminer la fin de l'adolescence, qui s'explique et se détermine par des facteurs sociaux et qui sont donc plus difficilement quantifiables et repérables (Cannard, 2015, p. 26). La fin de la scolarité de plus en plus tardive est un exemple des facteurs qui peuvent témoigner de la fin de la période d'adolescence (Coslin, 2017, p. 13; Habib et Cassotti, 2017, p. 383).

Parmi les facteurs individuels, les changements physiologiques reliés à l'adolescence sont, souvent, bien connus et, même, bien visibles. Ces changements sont marqués par le début de la poussée structurale et celui de la puberté (Cannard, 2015, p. 33). Par «poussée structurale», on entend une croissance accélérée ainsi qu'une prise de poids de la personne pendant la période d'adolescence (Godefroid, 2008, p. 702). La personne se métamorphose des points de vue morphologique et physique: les parties du corps ne se développent pas toutes en même temps, ce qui fait que le développement corporel ne se fait pas toujours de manière harmonieuse (Cannard, 2015, p. 36; Godefroid, 2008, p. 702). Ces changements physiques se déroulent généralement sur trois ans et se terminent vers 17 ans chez la fille et vers 21 ans chez le garçon (Godefroid, 2008, p. 702). La puberté qui débute entraîne des variations hormonales comme l'activation de la fonction hypothalamo-hypophysogonadique (Habib et Cassotti, 2017, p. 383). En latence pendant l'enfance, et ce, depuis la naissance, les hormones de l'hypothalamus, de l'hypophyse et des gonades se déclenchent soudainement à la puberté (Cannard, 2015, p. 32-33). Les caractères physiques propres à chaque sexe apparaissent (la voix se transforme, la barbe pousse) et le goût pour l'exploration des sentiments et des liens amoureux apparaît (Godefroid, 2008, p. 702)

Ces changements physiques, qui peuvent d'ailleurs apparaître soudainement, ont des répercussions dans la perception que l'adolescent peut avoir de l'image de son corps (Coslin, 2017, p. 43). L'adolescent voit son corps changer et doit intégrer en lui-même cette idée que son corps se modifie et actualiser la représentation qu'il a de son corps. Cette prise de conscience du corps est parée au regard des autres qui est de plus en plus important, et il arrive que cette prise de conscience entraîne un mal-être pouvant aller jusqu'à de l'anorexie (Coslin, 2017, p. 42).

Du point de vue cognitif, lors de la période de l'adolescence, une maturation cérébrale est constatée, qui se reflète par un décalage entre les régions contrôlant les processus cognitifs et les régions dédiées au traitement affectif (Habib et Cassotti, 2017, p. 385). La personne a alors la capacité d'utiliser des opérations formelles et de formuler des pensées abstraites plus complexes (Gibbons et Poelker, 2019, p. 196). En milieu d'adolescence, les capacités cognitives deviennent similaires à celles des adultes et se remarquent par leurs capacités à la prise de décision et à l'estimation des probabilités (Habib et Cassotti, 2017, p. 384). Pendant cette période, la personne découvre les lois qui régissent la société et le monde qui l'entoure, ce qui lui permet de développer sa capacité de raisonnement et sa capacité à établir des liens entre des notions abstraites (Godefroid, 2008, p. 711-717). L'adolescent est capable de faire référence à des concepts (Godefroid, 2008, p. 712).

La dimension sociale est un élément très important dans le stade de vie de l'adolescent. Parmi les facteurs sociaux, l'adolescence est marquée par les apprentissages sociaux et culturels lors desquels la personne commence à explorer les rôles sociaux, cette exploration au cours de laquelle elle choisira comment elle se conformera aux rôles ou non, les 
intégrera ou non (Coslin, 2017, p. 16). Pendant l'adolescence, la personne redéfinit et construit son identité. Elle explore de nouvelles possibilités s'ouvrant à elle et les nouveaux choix auxquels elle doit et peut faire face (Godefroid, 2008, p. 739). Les amis deviennent de plus en plus importants dans la vie des adolescents. Remplaçant en partie la place que les parents tenaient lors de l'enfance, les amis et les pairs deviennent des personnes plus significatives pendant l'adolescence et deviennent des agents socialisants importants (Godefroid, 2008, p. 739). Cette situation et ce positionnement personnel face aux rôles sociaux ont des impacts sur la construction de l'identité et la construction de soi de l'adolescent (Coslin, 2017, p. 16). La distanciation relationnelle avec leurs parents, quant à elle, prépare et permettent aux adolescents de prendre une plus grande autonomie.

Du point de vue affectif, l'adolescence est une période où la personne intègre les expériences passées qu'elle a vécues pendant son enfance, où elle fait le point sur sa vie, passée et future, et où s'amorce souvent une réflexion sur ses futures occupations et la vie professionnelle (Godefroid, 2008, p. 739). Son identité se construit aussi lorsqu'elle côtoie ses pairs et cherche à se faire accepter d'eux et du groupe auquel elle désire appartenir. Cette recherche identitaire peut mener l'adolescent à faire face à des difficultés qui peuvent mener à une diffusion de l'identité, c'est-à-dire la confusion de l'identité personnelle et des rôles qu'il peut être amené à jouer dans les différentes sphères de sa vie, dans ces dimensions affectives et sociales (Godefroid, 2008, p. 739). C'est le moment également où la personne cherche à explorer de nouvelles expériences. L'adolescence est souvent le moment d'adoption de comportements à risque à la recherche de sensations fortes (Habib et Cassotti, 2017, p. 383).

\section{Passage de vie}

La préadolescence et l'adolescence se distinguent des autres périodes de la vie. Il s'agit de moments d'introspection où la personne se découvre, fait de nouvelles expériences et se construit une identité qui lui est propre, qui l'amène et la prépare au monde adulte. Le préado et l'ado, de plus en plus intéressés par le monde extérieur, se détachent tranquillement du noyau familial et de ses activités. Les amis et les pairs deviennent très importants.

Bien que de nombreuses ressemblances existent, il y a des nuances importantes à prendre en compte entre ces deux périodes de vie. Le préadolescent vit ses premières expériences «de grand»; il reste cependant attaché au monde de l'enfance qu'il n'a pas encore quitté totalement. L'adolescent vit et établit une coupure avec ses parents, il explore le monde adulte sans tout à fait endosser les responsabilités, les droits et les avantages qui accompagnent l'âge de la majorité.

Dans les pratiques de lecture et les pratiques culturelles, on remarque trois effets d'âge, c'est-à-dire trois pratiques qui semblent être reliées, du point de vue statistique, à la période de développement de l'enfance et de l'adolescence. Premièrement, par la lecture des statistiques présentées précédemment, on note une diminution de la fréquence des activités de lecture entre les fourchettes de 6 à 9 ans, de 9 à 12 ans et de 12 et 15 ans. Deuxièmement, toujours selon les statistiques, il est possible de remarquer une diminution de la pratique de la lecture par plaisir entre les fourchettes de 6 à 9 ans, de 9 à 12 ans et de 12 et 15 ans. Finalement, on dénote, sans surprise, une augmentation de la fréquence d'activités incluant les technologies numériques pendant les temps libres entre les fourchettes de 6 à 9 ans, de 9 à 12 ans et de 12 et 15 ans.

Avec l'augmentation de l'utilisation des technologies numériques, que ce soit par les formats (cellulaires, tablettes numériques) ou par les médias (Instagram, Fortnite), il serait intéressant et presque essentiel de connaître plus en détail les usages que les jeunes peuvent en faire. Il serait alors peut-être possible de transférer la connaissance de leurs goûts technologiques à la lecture numérique et à la conception d'applications ou d'interfaces numériques de lecture.

Il est nécessaire, pour faire une analyse en profondeur des pratiques de lecture, sur papier ou en numérique, des jeunes, de le faire de manière globale en tenant compte du contexte québécois. Après recherches, nous constatons qu'il y a un manque important d'études et principalement d'études longitudinales sur les pratiques de lecture et pratiques culturelles des enfants de moins de 16 ans au Québec, études qui nous permettraient de connaître davantage ces jeunes.

Après tout, connaître l'enfant, le jeune d'aujourd'hui, est essentiel pour préparer le lecteur et le citoyen de demain.

L'auteure remercie le Fonds de recherche du Québec - Société et culture (FRQSC) pour son soutien financier, ainsi que Catherine Fortier et Dominic Desaulniers, bibliothécaires à l'Université de Montréal, pour leur précieuse aide à la recherche bibliographique. 


\section{SOURCES CONSULTÉES}

Ashfaq, H. et Ansari, M. N. (2018). Impact of Online Reading on Skills of Professionals. Library Philosophy and Practice: 1-13.

Bibliopresto.ca. (2017). Rapport annuel 2015-2016. Repéré à biblio presto.ca/pdf/rapportannuel_2016_bibliopresto.pdf

Bibliopresto.ca. (2018). Rapport annuel 2017-2018. Repéré à biblio presto.ca/pdf/rapportannuel_2018_bibliopresto.pdf

Biernacki, R. (2007). Practice. Dans G. Ritzer (dir.), The Blackwell Encyclopedia of Sociology. Oxford, Royaume-Uni: John Wiley \& Sons. doi: 10.1002/9781405165518.wbeosp086

Bloch, H. (1997). Comportement. Dans Dictionnaire fondamental de la psychologie. Paris, France: Larousse.

Boyd, D. et Bee, H. (2017). L'enfance : les âges de la vie. Montréal, QC: ERPI sciences humaines.

Burleigh, D. (2017). Using Consumer Data to Illustrate How the Library Channel Drives Digital Sales. Publishing Research Quarterly, 33(4): 380-391. doi : 10.1007/s12109-017-9555-y

Campeau, R., Sirois, M. et Rheault, É. (2004). La perspective sociologique. Dans Individu et société. Montréal, QC: Gaëtan Morin, 4-44.

Cannard, C. (2015). Du concept de jeunesse au concept d'adolescence. Dans Le développement de l'adolescent ( $2^{\mathrm{e}}$ éd.). Louvain-la-Neuve, Belgique: De Boeck Université, 9-30.

Choi, M.-J., Kim, S.-H., Lee, S., Kwon, B. C., Yi, J. S., Choo, J. et Huh, J. (2017). Toward Predicting Social Support Needs in Online Health Social Networks. Journal of Medical Internet Research, 19(8). Repéré à search.proquest.com/docview/1951538619?accountid=12543

Coslin, P. G. (2017). Psychologie de l'adolescence (5 éd.). Clamecy, France: Armand Colin.

Coulangeon, P. (2010). Sociologie des pratiques culturelles. Paris, France: La Découverte.

Dawkins, R. (2017). Tools for jimmying experience: Conceptual speed dating on Facebook. First Monday, 22(11). Repéré à search. proquest.com/docview/1961790299 ?accountid=12543

Demont, É. (2009). Le développement humain. Dans La psychologie. Auxerre, France: Éditions Sciences humaines, 63-92. doi: 10.3917/ sh.demon.2009.01

Du Mays, D. et Bordeleau, M. (2015). Les activités sédentaires chez les jeunes: qui les pratique et quelle en est l'évolution depuis 2007 ? Zoom santé, Avril(50): 1-8. Repéré à www.stat.gouv.qc.ca/ statistiques/sante/bulletins/zoom-sante-201504.pdf

Eisenberg, A. F. (2007). Habitus/Field. Dans G. Ritzer (dir.), The Blackwell Encyclopedia of Sociology. Oxford, Royaume-Uni: John Wiley \& Sons. doi : 10.1002/9781405165518.wbeosh001

Ellis, D. (1989). A behavioural approach to information retrieval system design. Journal of Documentation, 45(3): 171-212. doi : 10.1108/ eb026843

Ferréol, G., Cauche, P., Duprez, J.-M., Gadrey, N. et Simon, M. (2011). Dictionnaire de sociologie (4e éd.). Paris, France: Armand Colin.

Fortier, J.-F. et Pizarro Noël, F. (2013). La sociologie de A à Z. Montréal, QC: Pearson.

Fröhlich, W. (1997). Comportement. Dans Dictionnaire de la psychologie. Paris, France: Librairie Générale Française.

Fulton, C. et Henefer, J. (2017). Information Practice. Dans Encyclopedia of Library and Information Sciences. Boca Raton, FL: CRC Press, 2162-2171.
Gibbons, J. L. et Poelker, K. E. (2019). Adolescent Development in a Cross-Cultural Perspective. Dans Cross-Cultural Psychology: Contemporary Themes and Perspectives. Hoboken, NJ: Wiley Blackwell, 190-215.

Godefroid, J. (2008). Le développement de la personne. Dans Psychologie: sciences humaines et cognitives. Louvain-la-Neuve, Belgique: De Boeck Université, 692-747.

Gouvernement du Québec. (2019). Banque de données des statistiques officielles sur le Québec. Repéré à www.bdso.gouv.qc.ca

Habib, M. et Cassotti, M. (2017). La prise de risque à l'adolescence. Dans Psychologie du développement. Amsterdam, Pays-Bas: Elsevier Masson, 383-390.

Jansen, B. J. et Rieh, S. Y. (2010). The seventeen theoretical constructs of information searching and information retrieval. Journal of the American Society for Information Science and Technology, 61(8): 1517-1534. doi: 10.1002/asi.21358

Keenan, T., Evans, S. et Crowley, K. (2016). An introduction to child development. Los Angeles, CA : Sage Publications.

Kleijnen, E., Huysmans, F., Ligtvoet, R. et Elbers, E. (2017). Effect of a school library on the reading attitude and reading behaviour in non-western migrant students. Journal of Librarianship and Information Science, 49(3): 269-286. doi : 10.1177/0961000615622560

Late, E. (2018). Reading orientations in state research institutes. Information Research, 23(2). Repéré à search.proquest.com/docview/ 2078162223 ?accountid $=12543$

Lopatovska, I. et Sessions, D. (2016). Understanding academic reading in the context of information-seeking. Library Review, 65(8/9): 502-518. doi : 10.1108/LR-03-2016-0026

Magnan, R. (dir.) (2016). Les pratiques culturelles au Québec en 2014: Recueil statistique. Volume 1. Groupes sociaux. Québec, QC: Ministère de la Culture et des Communications. Repéré à www.mcc.gouv. qc.ca/fileadmin/documents/publications/Enquete_pratiques_ culturelles/RecueilStat2014-Vol1.pdf

Martel, M. D. (2017). Le design du «care» en bibliothèque: du tiers lieu au lieu d'inclusion sociale. I2D-Infoormation, données \& documents, 54(1): 52-54.

McGeown, S. P., Osborne, C., Warhurst, A., Norgate, R. et Duncan, L. G. (2016). Understanding Children's Reading Activities: Reading Motivation, Skill and Child Characteristics as Predictors. Journal of Research in Reading, 39(1): 109-125.

McKechnie, L. (2006). Spiderman is Not for Babies (Peter, 4 years): The "Boys and Reading Problem" from the Perspective of the Boys Themselves. The Canadian Journal of Information and Library Science, 30(1/2): 57-67.

Papalia, D., Olds, S. et Feldman, R. (2010). Le développement humain: vue d'ensemble et approches théoriques. Dans Psychologie du développement de l'enfant. Montréal, QC: Chenelière éducation, 2-35.

Papalia, D. et Martorell, G. (s. d.). Le développement humain: vue d'ensemble et approches théoriques. Dans Psychologie du développement humain. Montréal: Chenelière éducation, 3-39.

Poirier, C., Desjardins, M. K., Martet, S., Melançon, M.-O., Poirier, J., St-Germain-Blais, K. et Barrette, Y. (2012). La participation culturelle des jeunes à Montréal : des jeunes culturellement actifs. Montréal, QC: INRS Centre - Urbanisation Culture Société.

Reuter, K. (2007). Assessing aesthetic relevance: children's book selection in a digital library. Journal of the American Society for Information Science and Technology, 58(12): 1745-1763. doi: 10.1002/asi.20657 
Ross, C. S. (2006). Adult Readers. Dans Reading matters: what the research reveals about reading, libraries, and community. Westport, CT: Libraries Unlimited.

Rothbauer, P. (2006). Young Adults and Reading. Dans Reading matters: what the research reveals about reading, libraries, and community. Westport, CT: Libraries Unlimited.

Rutherford, L., Waller, L., Merga, M., McRae, M., Bullen, E. et Johanson, K. (2017). Contours of Teenagers' Reading in the Digital Era: Scoping the Research. The New Review of Children's Literature and Librarianship, 23(1): 27-46. doi: 10.1080/13614541.2017.1280351

Savolainen, R. (2007). Information behavior and information practice reviewing the "umbrella concepts" of information-seeking studies. Library Quarterly, 77(2): 109-132.

Savolainen, R. (2019). Modeling the interplay of information seeking and information sharing: A conceptual analysis. Aslib Journal of Information Management, 71(4) : 518-534. doi : 10.1108/AJIM-102018-0266
Scholastic. (2017). Kids and Family Reading Report, $7^{\text {th }}$ Edition. Repéré à www.scholastic.com/readingreport

Shaffer, D. et Kipp, K. (2014). Introduction of Developmental Psychology and its Research Strategies. Dans Developmental Psychology. Belmont, CA: Wadsworth, 3-43.

Vachon, H. (2010). La lecture. Dans R. Garon et M.-C. Lapointe (coord.), Enquête sur les pratiques culturelles au Québec (6éd.). Québec, QC: Ministère de la Culture, des Communications et de la Condition féminine, 13-40. Repéré à collections.banq.qc.ca/ark:/52327/ bs1968996

Wilson, T. D. (1999). Models in information behaviour research. Journal ofDocumentation, 55(3) : 249-270. doi : 10.1108/EUM0000000007145 